\begin{tabular}{|c|l|}
\hline Title & Existence or nonexistence of thermal pinning effect in grain growth under temperature gradient \\
\hline Author(s) & Ohno, Munekazu; Y amaguchi, Taichi; Sato, Dai suke; Matsuura, Kiy otaka \\
\hline Citation & $\begin{array}{l}\text { Computational Materials Science, 69, 7-13 } \\
\text { https://doi.org/10.1016/.Commatsci.2012.11.017 }\end{array}$ \\
\hline Issue Date & 201303 \\
\hline Doc URL & http://hdl.handle.net/2115/52230 \\
\hline Type & article (author version) \\
\hline File Information & CMS69_7-13.pdf \\
\hline
\end{tabular}

Instructions for use 


\title{
Existence or non-existence of thermal pinning effect in grain growth under temperature gradient
}

\author{
Munekazu Ohno, Taichi Yamaguchi, Daisuke Sato and Kiyotaka Matsuura
}

Division of Materials Science and Engineering, Faculty of Engineering, Hokkaido University, Kita 13 Nishi 8, Kita-ku, Sapporo, Hokkaido 060-8628, Japan

Corresponding author: Dr. Munekazu Ohno

Hokkaido University, Faculty of Engineering

Kita 13 Nishi 8, Kita-ku, Sapporo, 060-8628, Japan

Tel: +81-(0)11-706-6344

Fax: +81-(0)11-706-6344

E-mail adress: mohno@eng.hokudai.ac.jp

\begin{abstract}
:
In order to clarify existence or non-existence of the thermal pinning, viz., retardation of grain growth in polycrystalline materials due to steep temperature gradient, effects of the temperature gradient on the grain growth were systematically investigated by means of the phase-field simulations. It was found that the local grain growth is determined only by the local thermal history regardless of the temperature gradient and hence the temperature gradient does not directly influence the local grain growth. It was concluded that the thermal pinning does not exist when the grain boundary energy and mobility are isotropic and the grain growth is driven only by the reduction of total grain boundary energy.
\end{abstract}

Keywords:

Grain growth; Thermal pinning; Phase-field model; Steel; Welding; Casting; 


\section{Introduction}

The production of polycrystalline materials generally involves the grain growth under temperature gradient which is often steep in several production processes such as continuous casting and welding processes. Although considerable effort has been devoted to understanding of the grain growth kinetics [1-4], little has been revealed about effects of the temperature gradient on the grain growth.

It was found in a study on the heat affected zone (HAZ) in the fusion welding of a steel that the local grain size near the fusion line is smaller than the grain size predicted for the same thermal cycle as the HAZ but less temperature gradient [5]. It was speculated that the retardation of the local grain growth in the HAZ is ascribable to the steep temperature gradient. The temperature gradient in the weld HAZ is so steep that the temperature largely varies across a single grain. It is considered that the growth in higher temperature regions is hindered by that in the lower temperature regions because of the lower grain boundary mobility in the lower temperature regions. This might cause the retardation of the local grain growth and this phenomenon is termed the thermal pinning. According to this speculation, it is regarded that the essential factor causing the thermal pinning is the temperature dependence of the grain boundary mobility. It should be pointed out that in spite of its scientific and practical significance, the detail of the thermal pinning has not been examined yet. Most importantly, to the best of the authors' knowledge, there has been no experimental evidence directly verifying the existence of this effect, which should be because of the experimental difficulty in controlling very steep temperature gradient.

Recent progresses in computational materials science enable the detailed investigation of the grain growth during practical production processes of polycrystalline materials. Some Monte Carlo simulations on the grain growth in the HAZ demonstrated the existence of the thermal pinning [6-8]. However, the effects of temperature gradient on the grain growth were not systematically scrutinized and the detail of the thermal pinning remains to be clarified. In particular, it is not substantiated that the retardation of the local grain growth observed in these studies is entirely attributed to the temperature dependence of the grain boundary mobility. On the other hand, the Monte Carlo study by Holm et al. showed the contradictory finding that the temperature gradient does not affect the local grain growth kinetics under a fixed temperature distribution [9], which denies the existence of thermal pinning. It is therefore uncertain whether the thermal pinning actually exists or not.

The phase-field model has emerged as a powerful tool to simulate the microstructural evolution processes in a variety of phenomena based on the diffuse 
interface description [10-20]. This model can describe the grain growth in polycrystalline materials in real time and spatial scales. This is in contrast to the Monte Carlo simulation which generally involves the difficulty and/or uncertainty in relating the Monte Carlo step to the real time scale. One of the important features of the phase-field model is that the time evolution equation is exactly reduced to the equation for the curvature-driven motion of the grain boundary. This feature makes the relation between the theories of grain growth and the simulation outcome transparent [18]. In this study, the effects of temperature gradient on the grain growth are systematically investigated by means of the phase-field simulations.

The main concern of this study is the elucidation of the existence or non-existence of thermal pinning phenomenon. As mentioned above, the temperature dependence of the grain boundary mobility is of primary importance to the analysis on the thermal pinning. The system best suited for the present purpose is the one in which the grain growth is driven only by the reduction of the total amount of grain boundary energy and the grain boundary properties such as the grain boundary energy and mobility including their temperature dependences are isotropic. In this paper, we call this system quasi-ideal system, since the grain growth process in this system becomes equivalent to the ideal grain growth process when the temperature gradient vanishes. Our focus is placed on the quasi-ideal system in order to avoid any effect of secondary importance in the analysis. It should be noted that this system has not been subjected to the detailed investigation, since the earlier Monte Carlo studies [6-9] focused on the grain growth where the growth exponent characterizing the temporal change of the average grain size deviates from 0.5 of the ideal case.

\section{Computational details}

We employed the multi-phase-field model $[14,18]$ in which the grain structure is characterized by a set of phase-fields, $\left\{\phi_{i}(\mathbf{r}, t)\right\}$, each of which describes a probability of finding a grain $i$ at given point $\mathbf{r}$ and time $t$. The subscript $i$ specifies the type of grain, viz., the different crystallographic orientation and it is given by the integer number, the maximum number of which is denoted by $N . \phi_{i}(\mathbf{r}, t)$ takes 1 when the grain $i$ exists at given point $\mathbf{r}$ and time $t$ and it takes 0 otherwise. The grain boundary is then characterized as the region where $\phi_{i}$ continuously varies between 0 and $1 . \phi_{i}(\mathbf{r}, \mathrm{t})$ satisfies the normalization condition $\sum_{i}^{N} \phi_{i}(\mathbf{r}, t)=1$ at each spatial point. The time evolution of $\phi_{i}$ is described by the following equation [18] 


$$
\frac{\partial \phi_{i}}{\partial t}=-\frac{2 L}{n} \sum_{j \neq i}^{N} s_{i} s_{j}\left[\sum_{k \neq i}^{N}\left(\frac{\varepsilon^{2}}{2} \nabla^{2} \phi_{k}+\omega \phi_{k}\right)-\sum_{l \neq j}^{N}\left(\frac{\varepsilon^{2}}{2} \nabla^{2} \phi_{l}+\omega \phi_{l}\right)\right],
$$

where $L$ is the phase-field mobility, $s_{i}$ is a step function with $s_{i}=1$ for $\phi_{i}>0$ and $s_{i}=0$ otherwise, and $n$ represents the number of grains existing at a given spatial point. $\varepsilon$ is the gradient energy coefficient and $\omega$ is a constant. The quantities $\varepsilon$ and $\omega$ are related to the grain boundary energy $\sigma$ and the thickness of grain boundary $W$ as given by $\varepsilon=2 \sqrt{W \sigma} / \pi$ and $\omega=4 \sigma / W$. In this study, we focus on the grain growth in an austenitic steel. $\sigma$ is given by $\sigma=0.79 \mathrm{Jm}^{-2}$ [21] and $W$ is set to $6 \Delta x$ with the square grid spacing $\Delta x$ in the computational box. The time evolution equation of $\phi_{i}$ given by Eq. (1) exactly reproduces the curvature-driven motion of the grain boundary as described by $v$ $=-m \sigma \kappa$ where $v$ is the migration velocity of the grain boundary, $m$ is the grain boundary mobility and $\kappa$ is the mean curvature. The phase-field mobility $L$ is then related to $m$ as given by $L=m \sigma / \varepsilon^{2}$. In order to describe the effect of the temperature gradient on the grain boundary mobility, $L$ is made temperature-dependent by defining $L=$ $L_{0} \exp (-Q /(R T))$ with constants $L_{0}$ and $Q$ and the gas constant $R$. We employed $L_{0}=104$ $\mathrm{m}^{3} \mathrm{~J}^{-1} \mathrm{~s}^{-1}$ and $Q=177 \mathrm{~kJ} \mathrm{~mol}^{-1}$ based on the experimental results [22]. These values were chosen as typical values for the austenite grain growth in steels and we do not focus on a specific composition in this study.

We have carried out two-dimensional simulations, since the most essential kinetics causing the thermal pinning, stemming from the temperature dependence of grain boundary mobility, should be captured in the two-dimensional simulation. The computational box consists of 500 and 1000 square grid points for $x$ - and $y$-axes, respectively. In order to describe the coalescence-free growth, we employed the active parameter tracking algorithm proposed by Kim et al. [18]. In all the simulations, the temperature varies with $x$-coordinate and is uniform over $y$-axis. The periodic boundary condition was set along $y$-direction and zero flux boundary condition for $\phi_{i}$ was applied to the upper and lower edges in the $x$-direction. The square grid spacing $\Delta x$ was set to $\Delta x=4.0 \times 10^{-6} \mathrm{~m}$ and $\Delta t$ was given by $\Delta t=\Delta x^{2} /\left(5 L_{\max } \varepsilon^{2}\right)$ where $L_{\max }$ is the maximum value of $L$ under the temperature gradient.

\section{Results and discussions}

\subsection{Grain growth under a fixed temperature gradient}

We first focus on the grain growth under a fixed temperature gradient. The computational condition is as follows. The initial grain structure was obtained by 
solving Eq. (1) for an annealing period at $T=1743 \mathrm{~K}$ starting from small grains that were spatially distributed at random. Figure 1 shows an example of the initial grain structure. The initial average grain diameter $D_{\text {initial }}$ is $50 \mu \mathrm{m}$. The simulations were performed three times from three different initial grain structures with $D_{\text {initial }}=50 \mu \mathrm{m}$. The temperature varies across $x$-axis and the temperature gradient $d T / d x$ is negative. Its magnitude $G$ was set to $G=5.0 \times 10^{3}, 5.0 \times 10^{4}$ and $5.0 \times 10^{5} \mathrm{~K} \mathrm{~m}^{-1}$. The temperature at the center of $x$-axis $T_{c}$ was chosen to be $T_{c}=1273,1373$ and $1473 \mathrm{~K}$. The cooling rate $\dot{T}=-d T / d t$ was given as $\dot{T}=0.0,0.5,5.0$ and $50 \mathrm{~K} \mathrm{~s}^{-1}$. It is noted that the extremely high temperature regions appear when $G$ is as high as $5.0 \times 10^{5} \mathrm{~K} \mathrm{~m}^{-1}$. In reality, there should be a maximum temperature for the grain growth because of melting. Also, the extremely high temperature causes the large computational burden because very high grain boundary mobility at the high temperature requires very small time step. In order to avoid the computation for extremely high temperatures, the maximum temperature was set to $T=1743 \mathrm{~K}$ above which the temperature was assumed this value. Also, the minimum temperature was set to $T=973 \mathrm{~K}$ below which the temperature was assumed this value, since the grain growth does not virtually take place below this temperature. In the preliminary simulations, we confirmed that these procedures do not substantially alter the results shown below.

Figure 2 is an example of the time evolution of grain structure. This is the result for $T_{c}=1373 \mathrm{~K}, G=5.0 \times 10^{4} \mathrm{~K} \mathrm{~m}^{-1}$ and $\dot{T}=0.0$. The grain growth continuously occurs and the grains always have the equiaxed-shape. The grain size is slightly non-uniform in $x$-direction in each time period and it is relatively large on the left side, i.e., the high temperature side. The time evolution of the grain size is shown in Fig. 3. The grain size $D_{c}$ corresponds to the mean value in the center region of $x$-axis, which was estimated by first calculating the grain diameter at each $x$-coordinate based on the linear intercept method with the lines drawn parallel to $y$-axis and then by averaging them over $x$-coordinates of $\pm 20 \mu \mathrm{m}$ from the center of $x$-axis. Each plot represents the average of three runs and the maximum and minimum values are specified by the error bar of each plot. The results for three different values of $G$ and three different values of $T_{c}$ are shown. When the grains grow to be coarse, the error bar becomes large because the number of the grains counted in the averaging procedure becomes small. It is important to note that the results do not depend on $G$ for each value of $T_{c}$. The exception was observed for $T_{c}=1473$ and $1373 \mathrm{~K}$ when $G$ is as high as $5.0 \times 10^{5} \mathrm{~K} \mathrm{~m}^{-1}$. Under this steep temperature gradient, the grain size becomes coarser than the other cases in the late stage of process, as is opposite to what is expected from the thermal pinning effect. Figure 4 shows the snapshots of microstructure calculated for $T_{c}=1373 \mathrm{~K}$ and $G=$ 
$5.0 \times 10^{5} \mathrm{~K} \mathrm{~m}^{-1}$. It is seen that the grain size in the early stage is non-uniform due to the steep temperature gradient. As the holding time elapses, the large grains on the high temperature side grow toward the center part. These large grains grown from the high temperature side finally reach the center part at $t=100 \mathrm{~s}$, which results in the apparent acceleration of the grain growth in Fig. 3. This phenomenon should be regarded as the exceptional case, since this is not the normal grain growth, i.e., the competition of grains in the center region but the preferred and exclusive growth of the large grains from the high temperature edge (abnormal grain growth). Except for this phenomenon, the grain growth does not depend on $G$ for each value of $T_{c}$ in Fig. 3.

Hillert developed the theory of grain growth [1]. When the dependence of the grain boundary mobility on the temperature is explicitly specified, the time evolution of the average grain size obeys the following relation,

$$
\frac{d D}{d t}=\frac{M(T)}{D}
$$

where $D$ is the average grain size and $M(T)$ is given by $M(T)=M_{0} \exp (-Q /(R T))$ with a constant $M_{0}$ and the apparent activation energy for the grain growth $Q$. It should be noted that Eq. (2) does not consider the effect of temperature gradient and it describes the isothermal grain growth. In fact, we confirmed that the results of the phase-field simulations for $G=0$ (isothermal grain growth) are well reproduced by Eq. (2). The values of $M_{0}$ and $Q$ obtained from the present phase-field simulations are $M_{0}=0.0028$ $\mathrm{m}^{2} \mathrm{~s}^{-1}$ and $Q=177 \mathrm{~kJ} \mathrm{~mol}^{-1}$. The solid line in Fig. 3 is the calculated result of Eq. (2) for each value of $T_{c}$. All the results of the phase-field simulations for $G \neq 0$ accord well with the results of Eq. (2) for $G=0$. Therefore, it is evident from Fig. 3 that the temperature gradient $G$ itself does not influence the local grain growth. The local grain growth under a fixed temperature gradient is described as the time evolution of the grains growing isothermally at the local temperature. This is consistent with the finding in the earlier Monte Carlo study [10] and what we observed in the phase-field study on the discontinuous grain growth [23].

The temporal changes of the grain size in the center region during the cooling process are shown in Fig. 5. The initial temperature at the center of $x$-axis was set to $T_{c}$ $=1473 \mathrm{~K}$. The solid curve is the result of Eq. (2) calculated for each value of $\dot{T}$. The grain growth at each cooling rate is well described by Eq. (2). Although the data for $G=$ $5.0 \times 10^{4}$ and $5.0 \times 10^{5} \mathrm{~K} \mathrm{~m}^{-1}$ with $\dot{T}=0.5 \mathrm{~K} \mathrm{~s}^{-1}$ seem slightly lower than the solid line, the deviation falls within the range of the error bar. In fact, the results of two runs for these conditions are almost the same as the solid curve and only one result shows the lower value. From Figs. 3 and 5, it is understood that the temperature gradient $G$ does not 
influence the grain growth directly, but the local temperature and the local cooling rate essentially control the grain growth kinetics. In conclusion, the thermal pinning does not exist during the grain growth under a fixed temperature gradient in the quasi-ideal system.

\subsection{Grain growth under thermal cycle for $H A Z$}

The existence of the thermal pinning was suggested in the study on the HAZ of fusion welding process where the temperature profile is dependent on the time. The temperature profile in the HAZ can be calculated from the Rosenthal equation [24] which gives the temperature $T$ at a distance $x$ from the weld line moving at a velocity $v$ $\left(\mathrm{m} \mathrm{s}^{-1}\right)$ for a given energy input $q / v\left(\mathrm{~J} \mathrm{~m}^{-1}\right)$. Here $q$ is the arc power $\left(\mathrm{J} \mathrm{s}^{-1}\right)$. The spatial and temporal variations of the temperature in thin plates can be calculated by the following equation [25]

$$
T=T_{0}+\frac{q / v}{d \sqrt{4 \pi \lambda c t}} \exp \left(-\frac{x^{2}}{4 a t}\right),
$$

where $T_{0}$ is the initial temperature, $d$ is the thickness of the plate, $\lambda$ is the thermal conductivity, $c$ is the specific heat per unit volume and $a$ is the thermal diffusivity. The values of these quantities were set to $\lambda=41 \mathrm{Jm}^{-1} \mathrm{~s}^{-1} \mathrm{~K}^{-1}, c=4.5 \times 10^{6} \mathrm{Jm}^{-3} \mathrm{~K}^{-1}$ and $a=$ $9.1 \times 10^{-6} \mathrm{~m}^{2} \mathrm{~s}^{-1}$ based on the reported values for an austenitic steel [25]. The calculated result of Eq. (3) is shown in Fig. 6 for which $T_{0}=298 \mathrm{~K}, d=5 \mathrm{~mm}$ and $q / v=1.0 \times 10^{6} \mathrm{~J}$ $\mathrm{m}^{-1}$ were used. Figure 6(a) represents the thermal history and the time derivative of the temperature $d T / d t=-\dot{T}$ at fixed distances from the fusion line $\Delta x$. In this study, we define the position of the fusion line as the position where the maximum temperature during the thermal cycle reaches $T=1743 \mathrm{~K}$. It is seen that the cooling rate largely depends on the time and the position. Figure 6(b) shows the temperature gradient as a function of $\Delta x$ at the different time periods. The temperature gradient is almost spatially uniform but it gradually decreases with the time.

The solution of Eq. (3) is utilized to simulate the grain growth under the time-dependent temperature distribution. The origin of $x$-axis in the computational box for the grain structure was set to the position of fusion line. The temperature varies across $x$-direction and is uniform along $y$-direction. The initial grain structures, the boundary conditions and the averaging procedure of the grain size are the same as those used in the analysis in section 3.1. The temporal changes of the average grain sizes at $\Delta x$ $=0.16$ and $1.0 \mathrm{~mm}$ are shown in Fig. 7. The grain growth was assumed to start from $T=$ $973 \mathrm{~K}$. The plots represent the averaged values of three runs and the error bar indicates 
the maximum and minimum values of the three runs. Figure 7(a) is the result of $q / v=$ $1.0 \times 10^{6} \mathrm{~J} \mathrm{~m}^{-1}$. As is realized from Fig. 6(a), the region near the fusion line (i.e., small $\Delta x$ ) experiences the high temperature in the early period of the thermal cycle. Accordingly, the grain size at $\Delta x=0.16 \mathrm{~mm}$ is always larger than that at $\Delta x=1.0 \mathrm{~mm}$. The solid line is the result obtained at each $\Delta x$ by substituting the solution of Eq. (3) into Eq. (2) and by integrating it. Hence, this result represents the grain growth under the uniform temperature distribution $(G=0)$. One can see that the time evolutions of grain size obtained by the phase-field simulations for $G \neq 0$ accord well with those by Eq. (2) for $G=0$. We also tried $q / v=5.0 \times 10^{5} \mathrm{~J} \mathrm{~m}^{-1}$ to simulate the process for the different thermal cycle. The result is shown in Fig. 7(b). The grain growth behavior of phase-field simulations agrees with that by Eq. (2) in this case, too. Therefore, even in the processes under the time-dependent temperature distribution, the temperature gradient $G$ itself does not influence the local grain growth. Our simulations demonstrated that the thermal pinning phenomenon does not exist in the quasi-ideal system.

According to the speculation by Albery [5], the most essential factor causing the thermal pinning is considered the temperature dependence of the grain boundary mobility. Therefore, our attention in this study was directed at the system with the isotropic grain boundary properties and with no driving force other than the reduction of total grain boundary energy in order to avoid any effect of secondary importance to the thermal pinning. Contrary to the common thought, however, the present simulations indicated that the thermal pinning does not exist in this system, in other words, the existence of the thermal pinning cannot be explained only by the temperature dependence of the grain boundary mobility. There are several other possibilities yielding the retardation of local grain growth in the HAZ observed in the earlier study on the fusion welding [5]. For example, as the authors pointed out in their report [5], it may be ascribed to the difference in the grain boundary properties between the specimen used for the actual welding experiment and the simulation specimen used for the investigation of grain growth under small temperature gradient because these specimens have slightly different chemical compositions. Also, there may be the pinning effect of second phases stable at high temperatures such as the liquid phase. It is expected that the liquid phase exists even below the equilibrium solidus temperature near the fusion line in the HAZ, because of the non-equilibrium cooling process. The liquid may exist in the form of the grain boundary liquid and it causes a complete inhibition of the local grain growth. This possibility was discussed in the earlier Monte Carlo study [6] and it is investigated by the phase-field simulations in this study. 
In the simulations shown above, the region where the maximum temperature exceeds $T=1743 \mathrm{~K}$ was defined as the fusion zone and the grain growth was simulated in the region below this temperature. We now consider the (non-equilibrium) solidus temperature below $T=1743 \mathrm{~K}$ and the liquid phase pinning above the solidus temperature. All the simulation conditions are the same as those used for Figs. 6 and 7, except that the grain growth is completely inhibited when the temperature exceeds a given solidus temperature. The complete inhibition is described by setting the phase-field mobility to zero. We focus on three different solidus temperatures $T_{s}=1723$, 1693 and $1643 \mathrm{~K}$. The results for $q / v=1.0 \times 10^{6} \mathrm{~J} \mathrm{~m}^{-1}$ are shown in Fig. 8. The solid lines are the same curves as shown in Fig. 7(a). Although there is essentially no change in the grain growth at $\Delta x=1.0 \mathrm{~mm}$ (Fig. 8(b)), the retardation is clearly observed near the fusion line, viz., at $\Delta x=0.16 \mathrm{~mm}$ (Fig. 8(a)). The plateau in Fig. 8(a) indicates that the grain growth is completely inhibited in this time period during which the temperature is higher than the solidus. As the solidus temperature decreases, the grain size at $t=10 \mathrm{~s}$ decreases. Hence, the existence of the grain boundary liquid can be one of the possibilities that explain the retardation of local grain growth found in the study on the fusion welding. More detailed and quantitative analysis on this point remains as a future work.

\section{Summary}

In the previous study on the fusion welding, it was found that the grain size near the fusion line in the HAZ is smaller than that predicted for the same thermal cycle as the HAZ but less temperature gradient. It was supposed that the grain growth is inhibited by the steep temperature gradient because of the low grain boundary mobility in the low temperature region. This phenomenon is called the thermal pinning. In this study, in order to clarify the existence or non-existence of the thermal pinning, the effect of temperature gradient on the grain growth has been systematically investigated by means of the phase-field simulations. It was demonstrated that the thermal pinning does not exist in the system with the isotropic grain boundary properties and no driving force of the grain growth other than the reduction of total grain boundary energy. The present simulations indicated a possibility that the existence of the grain boundary liquid due to the non-equilibrium solidus temperature may retard local grain growth near the fusion line.

As mentioned above, the focus of this study was placed on the quasi-ideal grain growth process. There are several important factors that should be considered to describe the realistic grain growth process. For example, the solute segregation often 
exists at the grain boundary and it alters the grain growth because the kinetics is intimately connected to the diffusion process of the segregated atom(s) in this case. Also, the grain boundary properties are anisotropic in reality and the temperature dependence of local grain growth should accordingly be anisotropic. It is not clear how these factors affect the temperature dependence of grain growth. In this regard, we would like to refer to the previous works where the phase-field models were successfully extended to describe the grain growth with the solute segregation [26] and the anisotropic grain boundary properties [17]. Elucidation of effects of these factor remains as an important issue to be addressed to advance our understanding of the thermal pinning.

\section{Acknowledgements}

This work was partly supported by Grant-in-Aid for Young Scientists (A) (No. 2268067) from MEXT, Japan. 


\section{References}

[1] M. Hillert, Acta Metall. 13 (1965) 227-238.

[2] N.P. Louat, Acta Metall. 22 (1974) 721-724.

[3] F.J. Humphreys, Acta Mater. 45 (1997) 4231-4240.

[4] K. Matsuura, H. Sato, Y. Itoh and T. Narita, Tetsu-to-Hagané 78 (1992) 1488-1494.

[5] P.J. Alberry, B. Chew and W.K.C. Jones, Metals Technol. (1977) 317-325.

[6] B. Radhakrishnan and T. Zacharia, Metall. Mater. Trans. A 26A (1995) 2123-2130.

[7] S. Sista, Z. Yang and T. Debroy, Metall. Mater. Trans. B 32B (2000) 529-536.

[8] S. Mishra, T. DebRoy, Acta Mater. 52 (2004) 1183-1192.

[9] Y. Shi, D. Chen, Y. Lei, X. Li, Comput. Mater. Sci. 31 (2004) 379-388.

[10] E.A. Holm, N. Zacharopoulos and D.J. Srolovitz, Acta Mater. 46 (1998) 953-964.

[11] L.-Q. Chen, Annu. Rev. Mater. Res. 32 (2002) 113-140.

[12] N. Moelans, B. Blanpain, P. Wollants, Calphad 32 (2008) 268-294.

[13] D. Fan and L.-Q. Chen, Acta Mater. 45 (1997) 611-622.

[14] I. Steinbach and F. Pezzola, Physica D 134 (1999) 385-393.

[15] J.A. Warren, R. Kobayashi, E. Lobkovsky and W.C. Carter, Acta Mater. 51 (2003) 6035-6058.

[16] Y. Suwa, Y. Saito, H. Onodera, Acta Mater. 55 (2007) 6881-6894.

[17] N. Moelans, B. Blanpain and P. Wollants, Phys. Rev. Lett. 101 (2008) 025502-1-4.

[18] S.G. Kim, D.I. Kim, W.T. Kim, Y.B. Park, Phys. Rev. E 74 (2006) 061605-1-14.

[19] Y. Suwa, Y. Saito, H. Onodera, Comput. Mater. Sci. 40 (2007) 40-50.

[20] N. Moelans, F. Wendler, B. Nestler, Comput. Mater. Sci. 46 (2009) 479-490.

[21] E.D. Hondros, Proc. R. Soc. London A 286(1965) 479-498.

[22] C. Bernhard, J. Reiter and H. Presslinger, Metall. Mater. Trans. B 39B (2008) 885-895.

[23] M. Ohno, S. Tsuchiya and K. Matsuura, Metall. Mater. Trans. A 43A (2012) 2031-2042.

[24] D. Rosenthal, Trans. ASME 68 (1946) 849-866.

[25] M.F. Ashby and K.E. Easterling, Acta Metall. 30 (1982) 1969-1978.

[26] S.G. Kim and Y.B. Park, Acta Mater. 56 (2008) 3739-3753. 


\section{Figure caption list}

Fig. 1. Example of initial grain structure. The average grain diameter is $50 \mu \mathrm{m}$.

Fig. 2. Snapshots of microstructure during grain growth calculated for $T_{c}=1373 \mathrm{~K}, G=$ $5.0 \times 10^{4} \mathrm{~K} \mathrm{~m}^{-1}$ and $\dot{T}=0.0$.

Fig. 3. Temporal variations of grain diameter in the center region calculated for (a) $T_{c}=$ $1273 \mathrm{~K}$, (b) $T_{c}=1373 \mathrm{~K}$ and (c) $T_{c}=1473 \mathrm{~K}$. The cooling rate was set to $\dot{T}=0.0$ in all the cases. The square, circle and triangle plots are the simulation results for $G=5.0 \times 10^{3}$, $5.0 \times 10^{4}$ and $5.0 \times 10^{5} \mathrm{~K} \mathrm{~m}^{-1}$. These are the averaged values of three runs. The error bar in each plot indicates the minimum and maximum values. The solid line represents the calculated result of Eq. (2) for each value of $T_{c}$.

Fig. 4. Snapshots of microstructure during grain growth calculated for $T_{c}=1373 \mathrm{~K}, G=$ $5.0 \times 10^{5} \mathrm{~K} \mathrm{~m}^{-1}$ and $\dot{T}=0.0$.

Fig. 5. Temporal variations of grain diameter at the center position during cooling process from $1473 \mathrm{~K}$ at (a) $\dot{T}=0.5 \mathrm{~K} \mathrm{~s}^{-1}$, (b) $5.0 \mathrm{~K} \mathrm{~s}^{-1}$ and (c) $50 \mathrm{~K} \mathrm{~s}^{-1}$. The square, circle and triangle plots are the simulation results for $G=5 \times 10^{3}, 5 \times 10^{4}$ and $5 \times 10^{5} \mathrm{~K} \mathrm{~m}^{-1}$. These are the averaged values of three runs. The error bar indicates the minimum and maximum values. The solid lines represent the calculated results of Eq. (2).

Fig. 6. Temporal and spatial variations of temperature in the HAZ calculated by Eq. (3). (a) Thermal history and the cooling rate versus time. (b) Temperature gradient versus the distance from the fusion line $\Delta x$.

Fig. 7. Temporal changes of grain size at 0.1 and $1.0 \mathrm{~mm}$ away from the fusion line during the thermal cycle calculated from Eq. (3) for (a) $q / v=1.0 \times 10^{6} \mathrm{~J} \mathrm{~m}^{-1}$ and (b) $q / v=$ $5.0 \times 10^{5} \mathrm{~J} \mathrm{~m}^{-1}$. The solid lines are the results calculated by Eq. (2) with the solution of Eq. (3).

Fig. 8. Temporal changes of grain size at (a) $0.16 \mathrm{~mm}$ and (b) $1.0 \mathrm{~mm}$ away from the fusion line during the thermal cycle calculated from Eq. (3) for $q / v=1.0 \times 10^{6} \mathrm{~J} \mathrm{~m}^{-1}$. The solid lines are the results calculated by Eq. (2) with the solution of Eq. (3). 


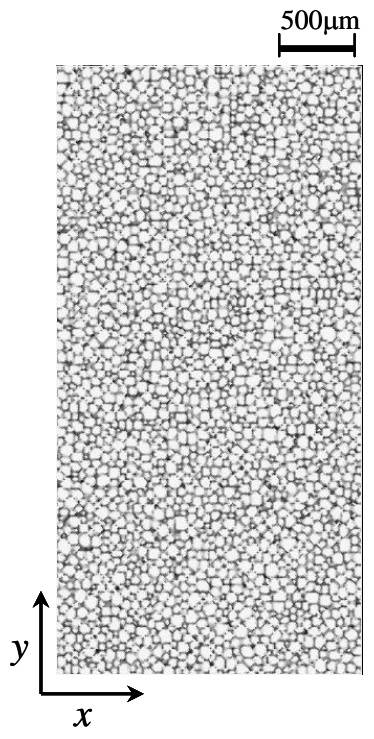

Fig. 1. Example of initial grain structure. The average grain diameter is $50 \mu \mathrm{m}$. 


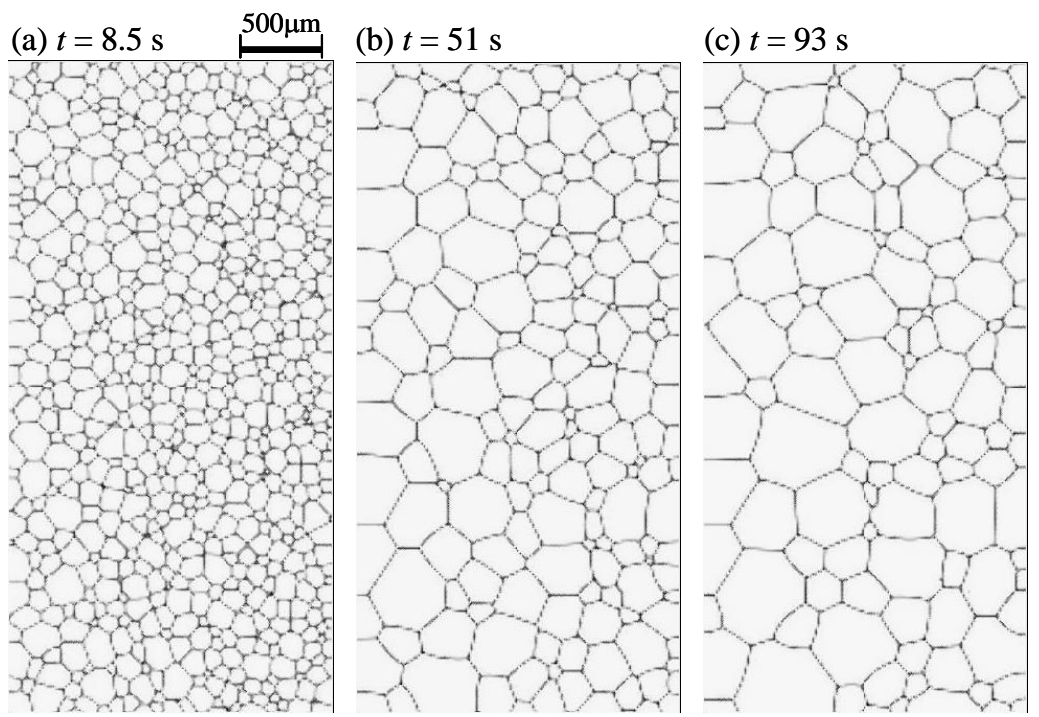

Fig. 2. Snapshots of microstructure during grain growth calculated for $T_{c}=1373 \mathrm{~K}, G=$ $5.0 \times 10^{4} \mathrm{~K} \mathrm{~m}^{-1}$ and $\dot{T}=0.0$. 
(a)

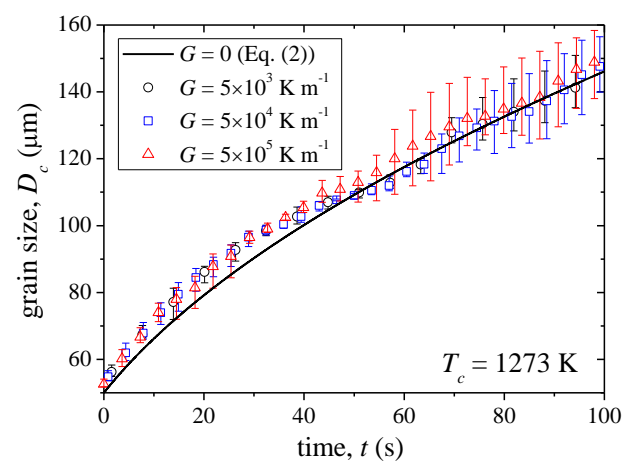

(c)

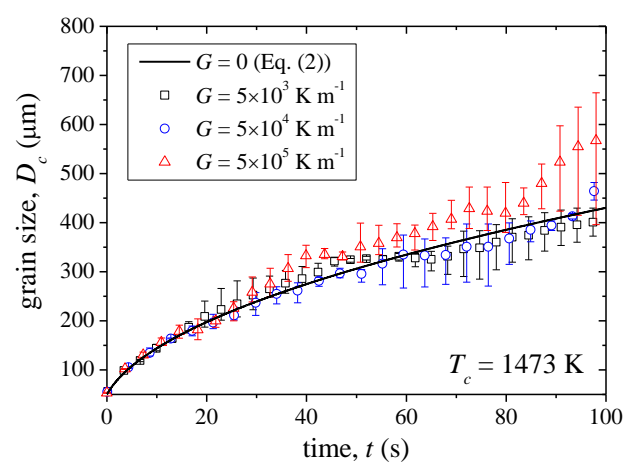

(b)

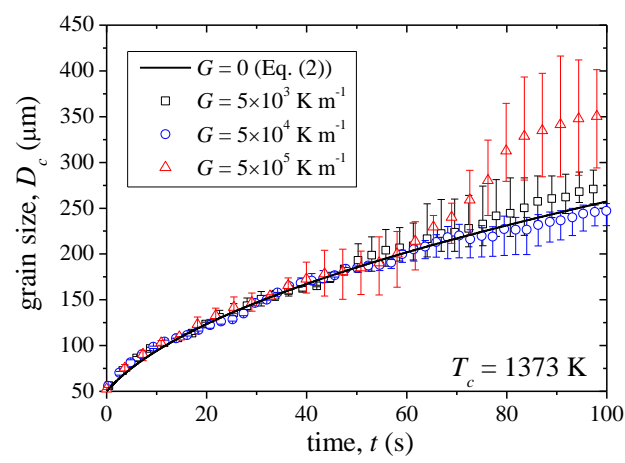

Fig. 3. Temporal variations of grain diameter in the center region calculated for (a) $T_{c}=$ $1273 \mathrm{~K}$, (b) $T_{c}=1373 \mathrm{~K}$ and (c) $T_{c}=1473 \mathrm{~K}$. The cooling rate was set to $\dot{T}=0.0$ in all the cases. The square, circle and triangle plots are the simulation results for $G=5.0 \times 10^{3}$, $5.0 \times 10^{4}$ and $5.0 \times 10^{5} \mathrm{~K} \mathrm{~m}^{-1}$. These are the averaged values of three runs. The error bar in each plot indicates the minimum and maximum values. The solid line represents the calculated result of Eq. (2) for each value of $T_{c}$. 


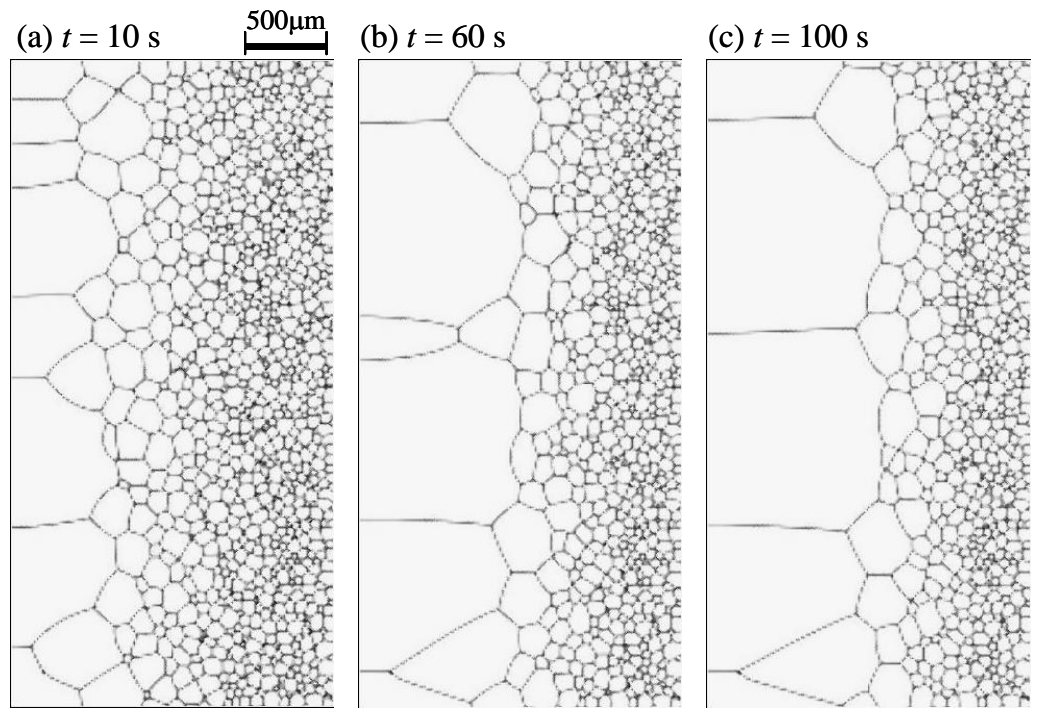

Fig. 4. Snapshots of microstructure during grain growth calculated for $T_{c}=1373 \mathrm{~K}, G=$ $5.0 \times 10^{5} \mathrm{~K} \mathrm{~m}^{-1}$ and $\dot{T}=0.0$. 
(a)

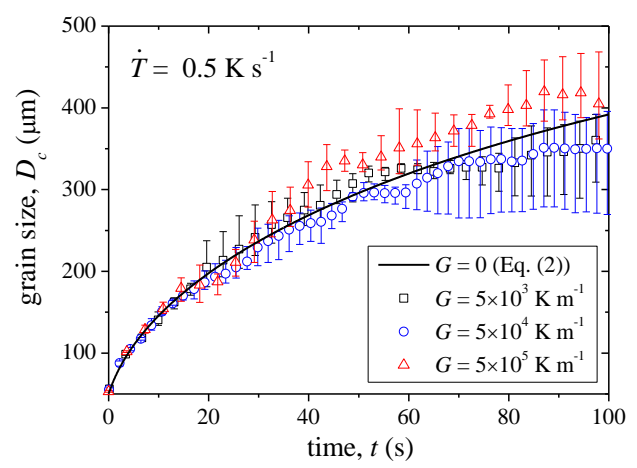

(c)

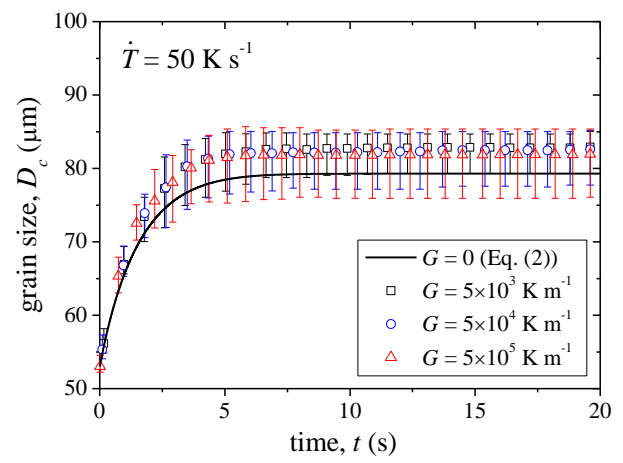

(b)

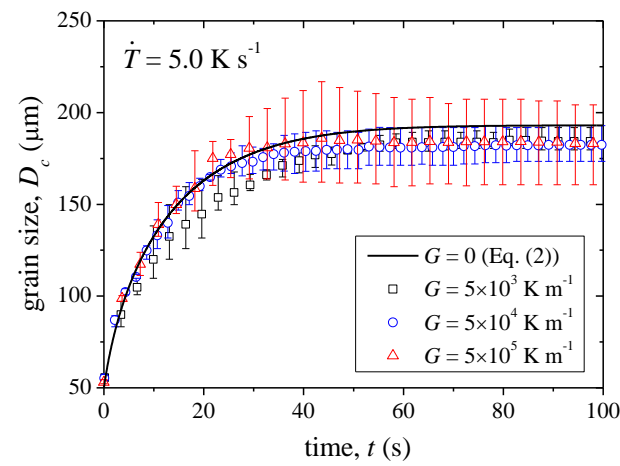

Fig. 5. Temporal variations of grain diameter at the center position during cooling process from $1473 \mathrm{~K}$ at (a) $\dot{T}=0.5 \mathrm{~K} \mathrm{~s}^{-1}$, (b) $5.0 \mathrm{~K} \mathrm{~s}^{-1}$ and (c) $50 \mathrm{~K} \mathrm{~s}^{-1}$. The square, circle and triangle plots are the simulation results for $G=5 \times 10^{3}, 5 \times 10^{4}$ and $5 \times 10^{5} \mathrm{~K} \mathrm{~m}^{-1}$. These are the averaged values of three runs. The error bar indicates the minimum and maximum values. The solid lines represent the calculated results of Eq. (2). 
(a)

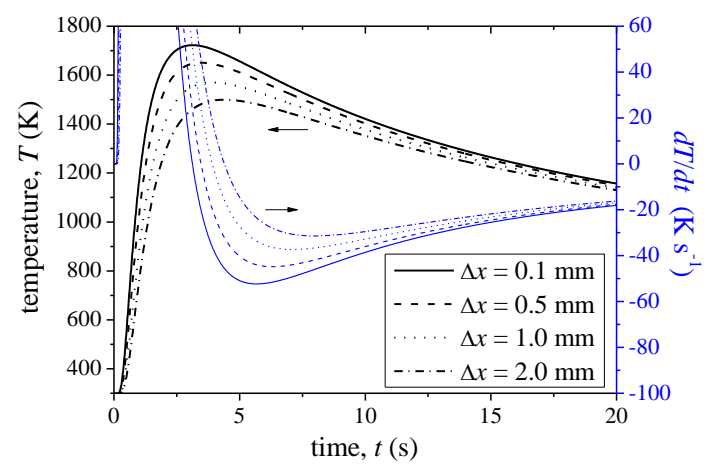

(b)

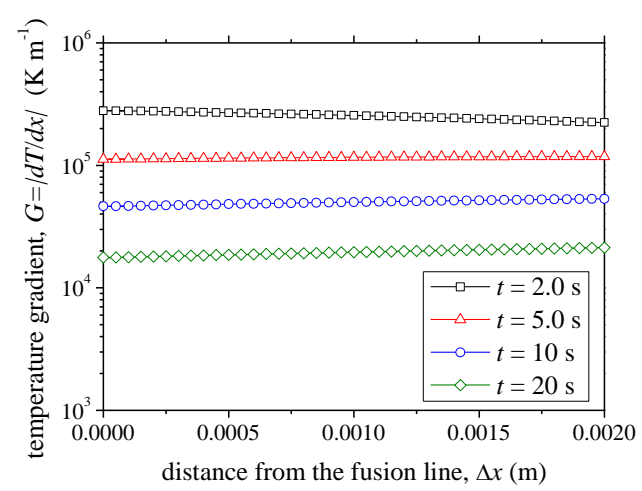

Fig. 6. Temporal and spatial variations of temperature in the HAZ calculated by Eq. (3). (a) Thermal history and the cooling rate versus time. (b) Temperature gradient versus the distance from the fusion line $\Delta x$. 
(a)

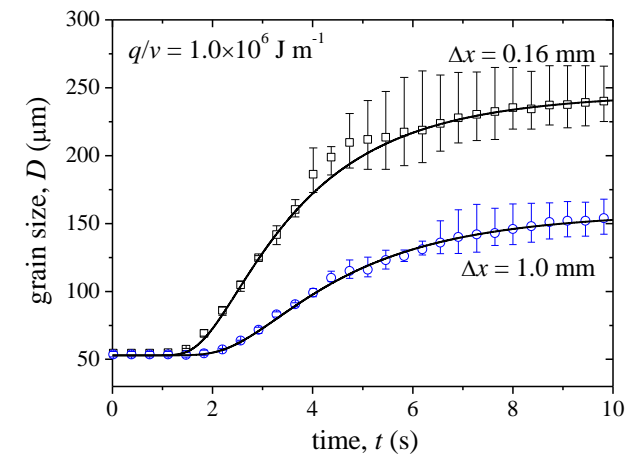

(b)

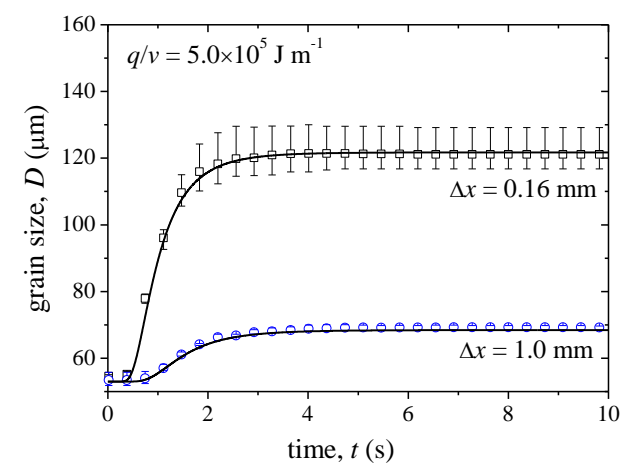

Fig. 7. Temporal changes of grain size at 0.1 and $1.0 \mathrm{~mm}$ away from the fusion line during the thermal cycle calculated from Eq. (3) for (a) $q / v=1.0 \times 10^{6} \mathrm{~J} \mathrm{~m}^{-1}$ and (b) $q / v=$ $5.0 \times 10^{5} \mathrm{~J} \mathrm{~m}^{-1}$. The solid lines are the results calculated by Eq. (2) with the solution of Eq. (3). 
(a)

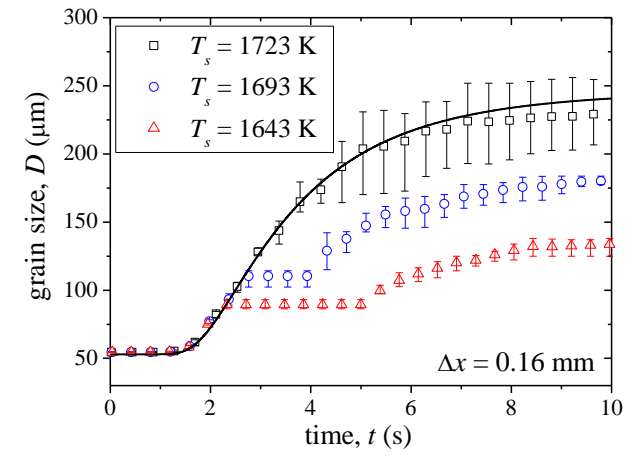

(b)

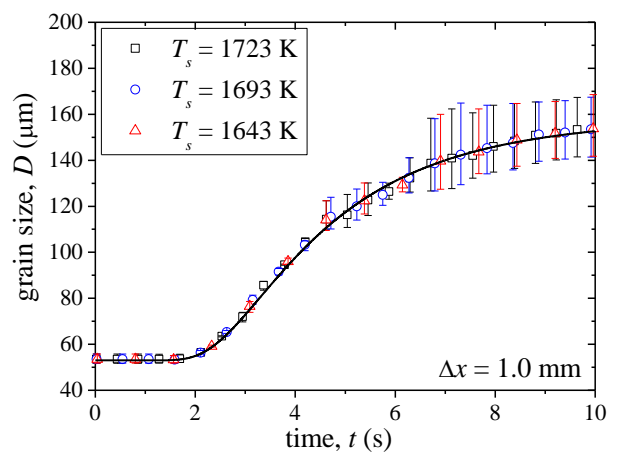

Fig. 8. Temporal changes of grain size at (a) $0.16 \mathrm{~mm}$ and (b) $1.0 \mathrm{~mm}$ away from the fusion line during the thermal cycle calculated from Eq. (3) for $q / v=1.0 \times 10^{6} \mathrm{~J} \mathrm{~m}^{-1}$. The solid lines are the results calculated by Eq. (2) with the solution of Eq. (3). 